\title{
APRESENTAÇÃO
}

\section{Funcionalismo(s) em Linguística: interface(s) entre morfossintaxe, semântica e pragmática na descrição e análise de língua(s)}

Functionalism (s) in Linguistics: interface(s) between morphosyntax, semantics and pragmatics in the description and analysis of language(s).

\author{
Flávia Bezerra de Menezes Hirata-Vale \\ (Universidade Federal de São Carlos) \\ Taísa Peres de Oliveira \\ (Universidade Federal de Mato Grosso do Sul/Três Lagoas)
}

Em termos gerais, considera-se que o paradigma funcionalista de estudo da linguagem se caracteriza por defender a inclusão das funções da linguagem no escopo da descrição linguística. No entanto, também se reconhece que sob o rótulo funcionalista, com Neves (2011), encontram-se diferentes correntes teóricas que se denominam, muitas vezes, a partir dos nomes daqueles que as propõem ou dos lugares onde são desenvolvidas. Assumindo uma perspectiva integradora, realizou-se em agosto de 2013, na Universidade Federal de São Carlos, o II Simpósio Internacional de Linguística Funcional - SILF, com o objetivo de promover a reunião de diferentes vertentes funcionalistas, propiciando um espaço para a discussão de suas premissas teóricas e procedimentos metodológicos, com destaque para suas aproximações e distanciamentos.

Os artigos que compõem este número temático da Revista DELTA são, portanto, oriundos de apresentações em conferências, 
mesas-redondas e simpósios temáticos realizados durante o II SILF, um conjunto de contribuições a partir do qual se pode vislumbrar o atual cenário da pesquisa em funcionalismo em diferentes regiões do Brasil, e também no exterior, e que comprovam a heterogeneidade dos estudos funcionalistas no país, e sua vocação para o estabelecimento de interfaces com áreas afins, tais como a Linguística Cognitiva e a Linguística Computacional. Faz-se, a seguir, uma breve descrição de cada um dos artigos deste volume.

Os dois primeiros artigos referem-se a duas conferências proferidas no simpósio, e têm, portanto, um caráter teórico mais abrangente.

O primeiro artigo refere-se à conferência apresentada por Daniel García Velasco, da Universidad de Oviedo, Espanha, em que tratou da Teoria da Gramática Discursivo Funcional (GDF), nos termos de Hengeveld e Mackenzie (2008). No artigo, Modularity and Derivation in Functional Discourse Grammar, o autor assume que a GDF é uma teoria modular no sentido proposto por Sadock (2012), uma vez que apresenta quatro níveis independentes de representação, cada qual com seus primitivos linguísticos. Nas gramáticas de cunho modular, essa relação entre os diferentes níveis, e mais especificamente, a natureza das interfaces, é uma questão central. Garcia Velasco considera que uma diferença crucial entre a GDF e outras abordagens modulares relacionase à noção de direcionalidade. Modelos como a Parallel Architecture, de Jackendoff $(1997,2002)$ são explicitamente descritos como nãodirecionais, enquanto a GDF é uma gramática de organização top-down, o que implica necessariamente que certos módulos não podem operar até que formulações pragmáticas e/ou semânticas tenham sido formuladas. $\mathrm{O}$ autor conclui que a GDF segue dois princípios básicos para sua implementação dinâmica: profundidade primeiro e profundidade máxima. Juntamente a restrições externas, esses princípios colaboram para criar representações linguísticas que são psicologicamente adequadas e possibilitam, se necessário, evitar níveis, simplificando as representações e permitindo seu não-alinhamento.

$\mathrm{O}$ artigo seguinte, de autoria de Maria Helena de Moura Neves (UNESP/UPM) intitula-se $A$ interface sintaxe, semântica e pragmática no Funcionalismo. Nesse texto, a autora traça um panorama de propostas funcionalistas de diferentes vertentes, a partir da verificação 
dos princípios funcionalistas que nelas estariam em relevância, nos diferentes casos. Para Neves, a partir desse panorama evidencia-se como cada proposta trata de modos distintos determinados temas e objetos de análise e, mais especificamente, como se estabelecem suas conexões particulares com outros campos de conhecimento. Nesse sentido, a autora aponta as semelhanças e as especificidades no tratamento da relação entre sintaxe, semântica e pragmática em diferentes perspectivas teóricas recentes do funcionalismo em linguística.

$\mathrm{Na}$ sequência, encontram-se trabalhos que tratam de questões teóricas mais específicas e que foram apresentados em diferentes simpósios temáticos e mesas redondas, relativos às distintas correntes funcionalistas praticadas no Brasil.

O artigo de Torrent, Lage, Tavares e Laviola, da Universidade Federal de Juiz de Fora, Minas Gerais, relaciona, de um modo bastante significativo, as contribuições da Linguística Cognitiva ao domínio da Linguística Computacional. Intitulado Relações de herança entre construções e entre frames: desafios da extensão do modelo construcionista para o domínio computacional no âmbito da FrameNet Brasil, o artigo discute as implicações de estender para o domínio computacional, no âmbito da FrameNet Brasil, os postulados teórico-analíticos das Gramáticas das Construções de Berkeley (Kay \& Fillmore, 1999; Fillmore, 2013) e Cognitivista (Goldberg 1995; 2006), em especial no que tange aos diferentes conceitos de herança construcional, ao Princípio da Motivação Maximizada e à interação entre frames verbais e construcionais. Em específico, os autores investigam se as relações de herança entre construções - pareamentos de uma forma evocadora de um frame-modeladas computacionalmente em um Constructicon, podem ser postas em paralelo como aquelas relações estabelecidas entre os frames na base de dados da FrameNet Brasil. A partir de sua análise, os autores concluem que é necessário modelar computacionalmente tanto a Herança Completa como outras formas de Herança Normal na base de dados do Constructicon, e, ao mesmo tempo, revisitar as relações entre frames propostas pela Berkeley FrameNet.

$\mathrm{O}$ artigo Mecanismos utilizados pelos destinatários do discurso para identificação de relações de coerência não sinalizadas por conectores, de Juliano Desiderato Antonio, docente da Universidade 
Estadual de Maringá, Paraná, toma como base a perspectiva da Teoria da Estrutura Retórica, uma teoria descritiva que tem por objeto o estudo da organização dos textos, caracterizando as relações que se estabelecem entre as partes do texto (Mann, Matthiessen \& Thompson, 1992; Mann \& Thompson, 1988; Matthiessen \& Thompson, 1988). Antonio objetiva, em seu texto, analisar outros mecanismos de sinalização das relações retóricas (ou relações de coerência), além de conectores utilizados pelos destinatários do discurso para identificação das relações. Sua investigação foi realizada a partir da apresentação de dez excertos para professores de curso superior, nos quais as relações retóricas não são sinalizadas por conectores, mas por outros meios, como pontuação, correlação modo-temporal, conteúdo das porções textuais, paralelismo sintático, paráfrase, inserção parentética, repetição, apresentação de evidências etc. Desse modo, o autor procurou verificar se a falta de sinalização por meio de um conectivo impediria ou dificultaria a identificação da relação e também se os informantes saberiam explicitar outros meios além dos conectivos que servissem como pista para identificação da relação. Antonio concluiu que, em sua grande maioria, as relações retóricas foram identificadas pelos informantes. As pistas indicadas pelos informantes foram formais, fonológicas, morfossintáticas, semânticas, textuais e cognitivas.

Adotando a perspectiva teórica da Linguística Centrada no Uso (Furtado da Cunha, Bispo \& Silva, 2013), Angélica Furtado da Cunha, da Universidade Federal do Rio Grande do Norte, compara em seu artigo, intitulado As Construções de movimento causado e ditransitiva: elos de polissemia, duas construções de estrutura argumental do português brasileiro - as construções de movimento causado (CMC) e ditransitiva (CD) - para investigar seus elos de polissemia, com base em aspectos sintáticos, semânticos e pragmáticos que elas possam compartilhar. A autora conclui que, para o português do Brasil, com base nos dados analisados, pode-se dizer que ambas as construções descrevem um evento de transferência: de lugar e de posse, respectivamente. Como a transferência de lugar parecer ser cognitivamente mais saliente, em termos perceptuais, a CD pode ser interpretada como uma extensão metafórica da CMC.

Ainda sob a perspectiva teórica da Linguística Centrada no Uso, Campos, Cezário e Alonso, da Universidade Federal do Rio de Janeiro, 
apresentam uma análise da formação da construção Xmente (como rapidamente e felizmente) no português. As autoras consideram em seu artigo o modelo da construcionalização/mudança construcional, conforme proposto por Traugott e Trousdale (2013), e procuram explicar tanto a formação de novos pareamentos simbólicos de forma e função (novos signos em rede na língua) como as mudanças de diferentes ordens que possam ocorrer com as construções já formadas. Campos, Cezário e Alonso postulam que o aumento da frequência de uso da estrutura Adjetivo+mente ocorreu no latim vulgar, na língua falada, que foi, por conseguinte, o berço da mudança. Nesse sentido, concluem que as mudanças não acontecem somente com -mente, mas com o contexto adjetivo + mente, iniciando-se ainda no latim, o que significa dizer que a mudança ocorre de modo holístico neste contexto e atinge a forma e o conteúdo de ambos os elementos da construção. O aparecimento da nova construção Xmente leva a um aumento do paradigma de advérbios, que vai se expandir ainda mais posteriormente, com o surgimento de novos usos com valores temporais.

Marize Mattos Dall'aglio Hattnher, da Universidade Estadual Paulista/São José do Rio Preto, examina a relação entre evidencialidade e tempo em um conjunto de línguas nativas do Brasil. O artigo Evidential subtypes and tense systems in Brazilian native languages, ilustra a possibilidade de interação entre a Gramática Discursivo-Funcional e os estudos tipológicos. A autora parte de uma classificação prévia (Hattnher, 2013), que lida exclusivamente com a relação entre o tempo e a expressão da evidencialidade por meio da percepção de eventos e da dedução, e a expande, levando em consideração, agora, quatro subtipos evidenciais, identificados em Hengeveld e Hattnher (2015). Mais especificamente, Hattnher objetiva mostrar que a natureza do processo mental envolvido na construção do significado evidencial determina a sua combinação com diferentes dimensões do passado, presente e futuro. A análise dos dados da amostra de línguas brasileiras nativas mostra que são poucas as restrições absolutas em relação à combinação entre evidencialidade e tempos específicos. Assim, as diferenças no ponto de referência utilizado para localizar o intervalo temporal relacionado a cada tempo aumentam a complexidade da relação entre evidencialidade e tempo. Além disso, a autora considera que a maioria das combinações que parecem ser desviantes é facilmente explicada se essas diferenças na organização da referência temporal forem levadas 
em conta, uma vez que o tempo associado à evidencialidade pode se referir apenas ao momento de ocorrência do evento descrito ou pode se referir ao momento em que o falante acessa a evidência relacionada ao evento que ele/ela descreve. Por fim, Hattnher encerra sua extensa e rica discussão argumentando que, embora se encontre variação nos sistemas temporais das línguas representadas no corpus, o tratamento teórico da GDF sobre a evidencialidade permite estabelecer algumas regularidades, no sentido de que se pode dizer que, nas línguas da amostra, é a semântica da evidencialidade que determina as opções de temporais.

À luz de desenvolvimentos recentes da Teoria dos Espaços Mentais (Sanders, Sanders \& Sweetser 2009, 2012; Ferrari \& Sweetser 2012), Diogo Pinheiro e Lilian Ferrari, da Universidade Federal do Rio de Janeiro, apresentam uma proposta de tratamento cognitivista do fenômeno da inversão do sujeito no português brasileiro (PB). Os autores argumentam que a inversão do sujeito está associada, no PB, à habilidade cognitiva de deslocamento do ponto de vista, ou seja, à categoria conceptual de perspectiva. Nesse sentido, os autores consideram que as estruturas SV e VS evocam redes distintas de espaços mentais: a primeira instrui o ouvinte a posicionar o Ponto de Vista no Centro Dêitico da Comunicação, ao passo que a segunda sinaliza o deslocamento do Ponto de Vista para o Domínio do Conteúdo. Pinheiro e Ferrari concluem que sua proposta se diferencia de outras abordagens a respeito do tema, uma vez que se valem, em sua análise, de uma categoria conceptual, de modo a determinar os processos cognitivos subjacentes à construção do significado das sentenças com sujeito pré e pós-verbal, e não, primariamente, de parâmetros formais (monoargumentalidade, inacusatividade, peso fonético, etc.) ou de parâmetros funcional-discursivos (acessibilidade referencial, topicalidade, etc.).

O artigo seguinte, intitulado Dêixis, espaços mentais e categorização: o caso dos pronomes we e you em inglês, de autoria de Helen de Andrade e de Lilian Ferrari, da Universidade Federal do Rio de Janeiro, também se baseia no referencial teórico da Linguística Cognitiva, e adota a Teoria dos Espaços Mentais (Fauconnier 1994, 1997, Fauconnier \& Turner 2002), assim como as propostas cognitivistas de análise da dêixis de Rubba (1996) e Marmaridou (2000). As autoras objetivam descrever os diferentes usos dos pronomes pessoais we $\left(1^{\mathrm{a}}\right.$ pessoa do 
plural) e you ( $2^{\text {a }}$ pessoa do singular/plural) em inglês, em termos de graus de prototipicidade. Nesse sentido, demostram em sua análise que os usos prototípicos desses pronomes, ancorados no Modelo Cognitivo Idealizado da dêixis, permitem a construção de usos não prototípicos. Essa polissemia dos dêiticos de pessoa we e you do inglês se deve, segundo as autoras, a diferentes processos de mesclagem conceptual. Cada um desses processos leva ao surgimento de um significado para os pronomes, e, em seus dados, Andrade e Ferrari identificam quatro diferentes significados para o pronome we e quatro para o pronome you: prototípico, inclusivo, exclusivo e virtual. Como conclusão, as autoras consideram que os resultados de sua pesquisa podem contribuir para uma melhor compreensão de como o processo de mesclagem pode ser responsável pelo surgimento de polissemia para termos já existente e promovem novas possibilidades descritivas e explicativas para o estudo da dêixis em outras línguas.

Roberto Gomes Camacho, da Universidade Estadual Paulista/São José do Rio Preto, investiga, em seu artigo Alinhamento e Estratégias de Relativização, a hipótese de que a seleção entre as diferentes estratégias de relativização disponíveis na gramática do português é motivada por um grau maior ou menor de transparência entre forma e conteúdo, o que implica, correlativamente, grau maior ou menor de facilidade no processamento cognitivo. Por essa razão, o autor considera que os diferentes graus de transparência permitem postular uma ordenação hierárquica das estratégias em correspondência a diferentes graus de facilidade no processamento cognitivo. Essa hipótese, afirma Camacho, baseia-se no princípio postulado por Comrie (1989), segundo o qual se uma língua dispõe de uma estratégia mais explícita e de uma estratégia menos explícita para a construção de relativas, o tipo mais explícito seria empregado para relativizar as posições mais baixas e o tipo menos explícito, para relativizar as posições mais altas. Um conceito que tem muita afinidade com esse princípio é o de alinhamento, postulado por Hengeveld e Mackenzie (2008) para representar o modo como unidades pragmáticas e semânticas não hierarquicamente relacionadas se projetam sobre as unidades morfossintáticas e fonológicas; em outros termos, como o processo de codificação reflete as motivações do processo de formulação. Assim, Camacho conclui que quanto mais direta a relação entre referência e codificação morfossintática, tanto maior o grau de iconicidade e, portanto, de transparência. Sendo assim, afirma o autor, 
se, por um lado, a seleção de diferentes estratégias de relativização pode ser motivada pelo grau menor ou maior de transparência entre forma e conteúdo, por outro, o grau de transparência é diretamente proporcional à facilidade de processamento cognitivo.

Também adotando o modelo teórico da Gramática DiscursivoFuncional (Hengeveld \& Mackenzie, 2008), o artigo seguinte, de Joceli Catarina Stassi-Sé, da Universidade Federal de São Carlos e de Erotilde Goreti Pezatti, da Universidade Estadual Paulista/São José do Rio Preto, trata de orações introduzidas por como e se que, embora apresentem a forma de uma subordinada adverbial, não dependem formalmente de uma oração principal. Em sua análise, as autoras analisam dados do Córpus Projeto Português Falado - Variedades Geográficas e Sociais, e consideram que essas orações são Movimentos, já que constituem uma contribuição autônoma para uma interação em desenvolvimento e podem ser ou desencadear uma reação, tendo sempre um efeito perlocutório. Essas construções são usadas no monitoramento da interação, para reativar, na memória do interlocutor, informações dadas no discurso, e salvaguardar a face do Falante, como estratégia de preservação de face. Stassi-Sé e Pezatti consideram que as funções, Resgate e Salvaguarda, são codificadas, no Nível Morfossintático, pela conjunção como e pela conjunção se respectivamente, e ressaltam que essas mesmas conjunções também são usadas para marcar a subordinação adverbial no Nível Representacional. Acrescentam, ainda, que, fonologicamente, são bem marcadas por meio da prosódia. A partir disso, as autoras postulam a existência de um domínio discursivo, determinante para esse tipo de construção, hierarquicamente superior, em termos funcionais, aos domínios representacionais, morfossintáticos e fonológicos característicos da GDF. Stassi-Sé e Pezatti argumentam que essas construções se referem a um tipo de fenômeno que emerge no nível da organização do discurso, entre porções textuais e não entre orações e, desse modo, afirma que, sendo determinada no Nível Interpessoal, a dependência das construções em relação a porções textuais maiores acontece no âmbito do discurso, não no âmbito sentencial, o que aponta para uma subordinação com orientação discursiva.

O último artigo deste volume, de autoria de Taísa Peres de Oliveira, da Universidade Federal de Mato Grosso do Sul/Três Lagoas e Flávia Bezerra de Menezes Hirata-Vale, da Universidade Federal de 
São Carlos, recorre teoricamente a concepções funcional-cognitivistas, nos termos de Bybee (2010) e Dancygier (1998), para avaliar diferentes padrões de construções condicionais no português, a partir dos parâmetros de condicionalidade. Ao adotar esses pressupostos teóricos, as autoras consideram, portanto, que a gramática está assentada sobre o uma rede de construções e que o significado é relativamente instável e sujeito às determinações cognitivo-perceptuais e discursivas. Nesse sentido, suas reflexões partiram do princípio cognitivista mais básico de que uma categoria não pode estar organizada apenas em termos de esquemas prototípicos, mas também no entendimento de como elementos periféricos se relacionam ao núcleo definidor da categoria. Desse modo, Oliveira e Hirata-Vale tomaram os traços básicos da condicionalidade como parâmetros definidores para o entendimento das diferentes molduras sintáticas - estruturas iniciadas por se e caso; estruturas iniciadas por conectores condicionais complexos e estruturas marcadas por conectores de outros esquemas conceituais (temporais, aditivas e alternativas) - que podem expressar o significado de condição em português. As autoras consideram que a análise dos dados revelou a complexidade da condicionalidade como uma rede que abriga construções de natureza formal e funcional bastante variadas, e que, além disso, foi possível evidenciar quais os traços da condicionalidade, como causalidade, predição e postura epistêmica, aproximam os exemplares do núcleo prototípico, sendo portanto mais básicos, e quais encontram-se diluídos, distanciando os membros uns dos outros. As autoras concluem, portanto, que a reflexão principal a que se chega é que a consideração da condicionalidade não pode simplificar-se na descrição de seu núcleo prototípico, mas deve explicar também os efeitos que a acomodação de elementos com complexidade morfossintática tão diferenciada tem no significado condicional, considerando-se, ainda, de que modo os exemplares menos prototípicos contribuem com a categoria e como se relacionam com outros membros dessa categoria.

Como se vê, encontram-se representadas neste volume temático da Revista Delta as mais diversas correntes funcionalistas em atuação em nosso país, que congregam pesquisadores de diferentes grupos de pesquisa em Funcionalismo do Brasil. Cumpre-se, assim, com muito sucesso o objetivo inicial do II SILF, o de aproximar pesquisadores de diferentes vertentes funcionalistas, a fim de discutir questões teóricas e descritivas a partir de pontos de vista distintos, abordando, espe- 
cificamente a questão da interface entre morfossintaxe, semântica e pragmática na descrição e análise das línguas.

Como organizadoras, gostaríamos de agradecer a colaboração dos pareceristas ad hoc, nossos colegas que aceitaram gentilmente avaliar os artigos submetidos a este volume. Nosso reconhecimento também é devido à FAPESP (Processo 2013/11116-0) e à CAPES (AUX-PE-PAEP 1118/2013), que nos auxiliaram financeiramente para a realização do II SILF. Por fim, agradecemos imensamente à Revista DELTA, e a toda sua equipe, pela oportunidade de publicação deste número especial.

Recebido em dezembro de 2016

Aprovado em janeiro de 2017

E-mails: fbmhvale@gmail.com taisapoliveira@gmail.com

\section{Referências bibliográficas}

Bybee, J. 2010. Language, Usage and Cognition. Cambridge: Cambridge University Press.

Comrie, B. 1989. Language universals and linguistic typology. Oxford: Blackwell.

DANCYGIER, B. 1998. Conditionals and predication (Cambridge Studies in Linguistics). Cambridge: Cambridge University Press.

Fauconnier, G. 1994. Mental Spaces. Cambridge: Cambridge University Press.

1997. Mappings in Thoughts and Language. Cambridge: Cambridge University Press.

FAUCONNIER, G. \& TURNER, M. 2002. The way we think: conceptual blending and the mind's hidden complexities. New York: Basic Books.

FiLlmORE, C. J. 2013. Berkeley construction grammar. In: HOFFMANN, T. \& TROUSDALE, G. (eds.). The Oxford Handbook of Construction Grammar. New York: Oxford University Press.

Furtado da Cunha, M. A.; Bispo, E. B. \& Silva, J. R. 2013. Linguística funcional centrada no uso: conceitos básicos e categorias analíticas. In: CEZARIO, M. M. \& FURTADO DA CUNHA, M. A. (orgs.). Linguística centrada no uso: uma homenagem a Mário Martelotta. Rio de Janeiro: Mauad X/FAPERJ. p. 13-39. 
GoldBERG, A. E. 1995. Constructions: A construction grammar approach to argument structure. Chicago: Chicago University Press.

. 2006. Constructions at work: The nature of generalization in language. New York: Oxford University Press.

HatTNHER, M. M. D. A. 2013. The interaction between tense and evidentials of event perception and deduction in Brazilian Native languages. In: MACKENZIE, J. L. \& OLBERTZ, H. (eds.). Casebook in Functional Discourse Grammar. Amsterdam: John Benjamins, p. 39-66.

Hengeveld, K. \& Mackenzie, J. L. 2008. Functional Discourse Grammar. A typologically-based theory of language structure. Oxford: Oxford University Press.

Hengeveld, K. \& Hattnher, M. M. D. A. 2015. Four types of evidentiality in the native languages of Brazil. Linguistics, v.53, n.3, p. 479-524.

Jackendoff, R. J. 1997. The Architecture of the Language Faculty. Cambridge, Mass.: The MIT Press.

. 2002. Foundations of Language. Oxford: Oxford University Press.

KAY, P. \& Fillmore, C. J. 1999. Grammatical constructions and linguistic generalizations: The what's X doing Y construction. Language, 75: $1-34$.

Mann, W. C. \& ThOmPson, S. A. 1988. Rhetorical Structure Theory: toward a functional theory of text organization. Text, 8(3): 243-281.

Mann, W. C., C. Matthiessen \& S. A. Thompson. 1992. Rhetorical Structure Theory and text analysis. In: MANN, W. C. \& THOMPSON, S. A. (eds.). Discourse description: diverse linguistic analyses of a fund-raising text. Amsterdam: John Benjamins.

Marmaridou, S. 2000. Pragmatic Meaning and Cognition. Amsterdan/ Philadelphia: John Benjamins Publishing Company.

Matthiessen, C. \& S. A. Thompson. 1988. The structure of discourse and 'subordination'. In: HAIMAN J.; THOMPSON S. (eds.). Clause Combining in Grammar and Discourse. Amsterdam/Philadelphia: John Benjamins.

Neves, M. H. M. 2011. Gramática de usos do português. 2. ed. São Paulo: Ed. UNESP.

RubBa, J. 1996. Alternate grounds in the interpretation of deitic expressions. In: Spaces, worlds \& grammar. Chicago: University of Chicago Press. p. 227-261.

SAdock, J. A. 2012. The Modular Architecture of Grammar. Cambridge: Cambridge University Press.

Sanders, T; Sanders J. \& Sweetser, E. 2009. Causality, cognition and communication: a mental space analysis of subjectivity in causal 
33.1

2017

connectives. In: Ted SANDERS \& SWEETSER, E. (eds.). Causal categories in discourse and cognition. Berlin/New York: Mouton de Gruyter.

.2012. Responsible subjects and discourse causality: how mental spaces and perspective help identifying subjectivity in Dutch backwards causal connectives. Journal of Pragmatics, 44/2: 191213.

Traugott, E.C. \& Trousdale, G. 2013. Constructionalization and Constructional Changes. Oxford: Oxford University Press. 